\title{
Effects of particle size and composition on coercivity of Sm-Co nanoparticles prepared by surfactant-assisted ball milling
}

\author{
Narayan Poudyal, Chuan-bing Rong, and J. Ping Liu ${ }^{\text {a) }}$ \\ Department of Physics, University of Texas at Arlington, Arlington, Texas 76019, USA
}

(Presented 19 January 2010; received 30 October 2009; accepted 1 December 2009; published online 15 April 2010)

\begin{abstract}
The $\mathrm{SmCo}_{\mathrm{x}}(\mathrm{x}=3.5,4,5,6,8.5$, and 10) magnetic nanoparticles with different composition have been prepared by surfactant-assisted ball milling technique. By controlling the settle-down time and the centrifugation conditions, the $\mathrm{SmCo}_{\mathrm{x}}$ nanoparticles with different particle size and narrow size distribution were successfully obtained. It was observed that the SmCo nanoparticles become unstable with increasing $\mathrm{Sm}$ content. It was also observed that the coercivity of the $\mathrm{SmCo}_{\mathrm{x}}$ nanoparticles increases with Co content and particle size, indicating a complex effect of the particle size and composition on magnetic hardening of the hard magnetic nanoparticles. () 2010 American Institute of Physics. [doi:10.1063/1.3334460]
\end{abstract}

\section{INTRODUCTION}

Rare-earth transition-metal compounds based on the $\mathrm{Sm}-\mathrm{Co}$ and $\mathrm{Nd}-\mathrm{Fe}-\mathrm{B}$ intermetallic systems have very high magnetocrystalline anisotropy among all the known materials. ${ }^{1}$ Hard magnetic $\mathrm{Sm}-\mathrm{Co}$ and $\mathrm{Nd}-\mathrm{Fe}-\mathrm{B}$ nanoparticles have drawn a great deal of attention owing to potential applications of the nanoparticles in high performance nanocomposite permanent magnets and ultrahigh-density recording media. ${ }^{2-5}$ A series of physical and chemical preparation techniques have been employed to fabricate rare-earth containing magnetic nanoparticles. ${ }^{6-9}$ However, fabrication of rare-earth containing nanoparticles has been proven to be very challenging due to their high chemical instability. Recently, we reported preparation of hard magnetic Sm-Co and $\mathrm{Nd}-\mathrm{Fe}-\mathrm{B}$ nanoparticles by surfactant-assisted ball milling. ${ }^{8,9}$ In this work, we focused on the size and composition effect on coercivity of the Sm-Co nanoparticles.

\section{EXPERIMENTAL PROCEDURE}

The starting $\operatorname{SmCo}_{\mathrm{x}}(\mathrm{x}=3.5,4,5,6,8.5$, and 10) alloy powders were prepared by arc melting followed by grinding. The powders with a particle size less than $45 \mu \mathrm{m}$ were mixed with organic solvent heptane $(99.8 \%$ purity) and surfactants (90\% oleic acid and $98 \%$ oleylamine) for ball milling. The mixtures were milled for $20 \mathrm{~h}$ with balls made of hardened steel by using a Spex $8000 \mathrm{M}$ high-energy ball milling machine and the powder to ball weight ratio is $1: 10$. Handling of the starting materials and as-milled products was carried out in an argon gas environment inside a glove box to protect the particles from oxidation. A size selection process was employed after the milling process to obtain particles with different size by controlling time of sediment of the diluted solutions containing milled slurries, termed as "settle-down time" in this paper and by controlling centrifugal conditions (details in Sec. III). Magnetic properties were measured by an alternating gradient magnetometer and a su-

${ }^{\text {a)} E l e c t r o n i c ~ m a i l: ~ p l i u @ u t a . e d u . ~}$ perconducting quantum interference device magnetometer. Structural, morphological, and compositional characterizations were performed using x-ray diffraction (XRD), small angle $\mathrm{x}$-ray scattering (SAXS), laser particle size analyzer (LPSA), transmission electron microscope (TEM), scanning electron microscope (SEM), and energy dispersive x-ray analysis.

\section{RESULTS AND DISCUSSION}

The experiments started with $\mathrm{Sm}_{2} \mathrm{Co}_{17}$ materials. During the sediment process, the finest particles are floating-at-top of the solutions. Figures 1(a) and 1(b) show the TEM images of $\mathrm{Sm}_{2} \mathrm{Co}_{17}$ nanoparticles of the floating-at-top and the $3 \mathrm{~h}$ settle-down nanoparticles with particle size about $6 \mathrm{~nm}$ and $20 \mathrm{~nm}$, respectively. The SEM image of the slurry particles is also given in Fig. 1(c) for comparison. The $\mathrm{Sm}_{2} \mathrm{Co}_{17}$ nanoparticles with different size were obtained by the following procedures:

(i) The floating-at-top nanoparticles. The particles were milled for $20 \mathrm{~h}$ in presence of the surfactants and were then ultrasonically vibrated in heptane solvent before being settled-down for $24 \mathrm{~h}$, during which larger particles were sedimentated gradually while smaller particles were still floating in the solution. After this settle-down time, a centrifugal separation was per-

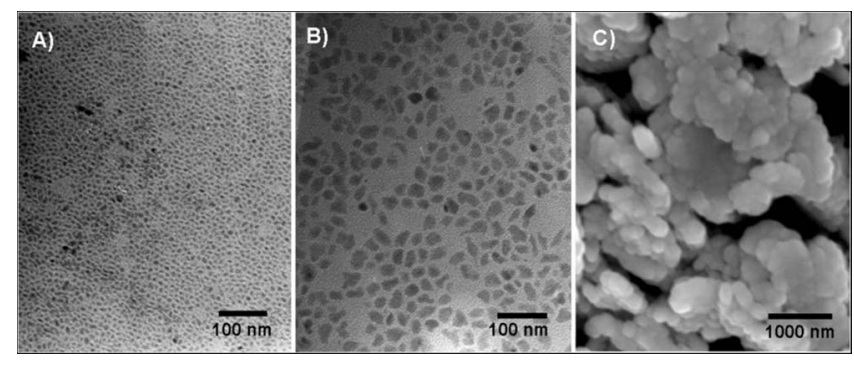

FIG. 1. TEM images of $\mathrm{Sm}_{2} \mathrm{Co}_{17}$ (a) floating-at-top nanoparticles $(\sim 6$ $\mathrm{nm})$, (b) nanoparticles collected from $3 \mathrm{~h}$ settled-down time $(\sim 20 \mathrm{~nm})$, and (c) SEM image of submicron $\mathrm{Sm}_{2} \mathrm{Co}_{17}$ slurry particles. 


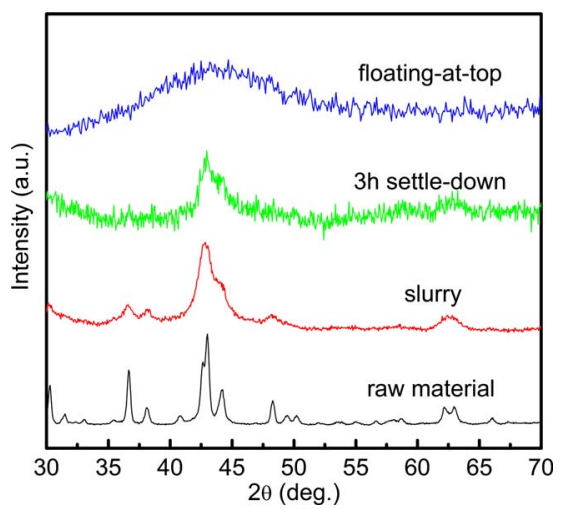

FIG. 2. (Color online) XRD patterns of the floating-at-top, $3 \mathrm{~h}$ settle-down, slurry particles, and starting powders.

formed to remove the large particles. The smallest nanoparticle size that could be obtained was $6 \mathrm{~nm}$.

The $3 \mathrm{~h}$ settle-down nanoparticles. The particles were milled for $20 \mathrm{~h}$ and were washed once in heptane to remove the finest floating nanoparticles. Then the remaining part was transferred into a centrifugal tube and dispersed in heptane again by similar ultrasonic vibration process, the dispersed solution was then settled-down for $3 \mathrm{~h}$. After this settling-down time, the particles floating in solvent were collected and a low-speed centrifugal separation was performed to remove the large particles.

(iii) The slurry particles. The as-milled product consists of particles with size ranging from several nanometers to submicron. The coarse particles, which sedimentate quickly in several minutes even when dispersed in heptane by similar ultrasonic vibration are referred as slurry particles.

It can be seen from Fig. 1 that both the 6 and $20 \mathrm{~nm}$ nanoparticles have narrow size distribution. The particle size and size distribution are also measured and confirmed by SAXS analysis (not shown here). However, the size of the slurry particles is in submicron range as shown in Fig. 1(c), indicating that the size selection process is effective in obtaining nanoparticles with controlled size and size distribution.

Figure 2 shows the XRD patterns of the floating-at-top, the $3 \mathrm{~h}$ settle-down nanoparticles, and the slurry $\mathrm{Sm}_{2} \mathrm{Co}_{17}$ nanoparticles obtained by the size selection process. For comparison, the XRD pattern of the starting $\mathrm{Sm}_{2} \mathrm{Co}_{17}$ powder is also shown here. It was found that the diffraction peaks of the $3 \mathrm{~h}$ settle-down nanoparticles and slurry particles are broadened, which means that the grain size is significantly reduced. However, it is difficult to quantitatively calculate the accurate grain size due to low diffraction intensity and the complexity of the strains and possible amorphorization induced during the ball milling process. As one can see that the floating-at-top nanoparticles only show one broad peak, due to the amorphorization in the very small nanoparticles. Importantly, no diffraction peaks from rare-earth oxides were found in the XRD patterns of all nanoparticles, indicating

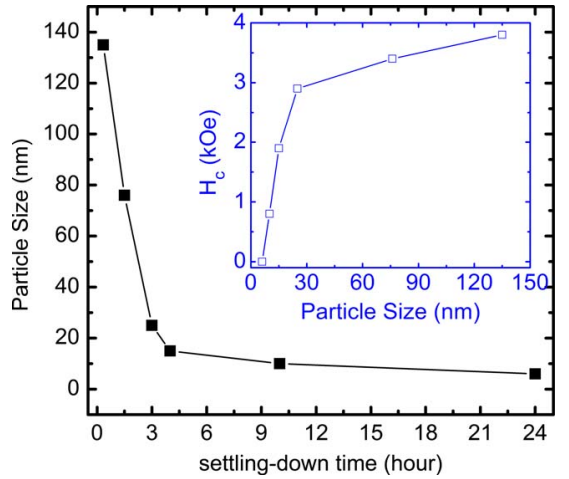

FIG. 3. (Color online) Dependence of particle size on settle-down time of $\mathrm{Sm}_{2} \mathrm{Co}_{17}$ nanoparticles. The inset gives the size dependent coercivity of $\mathrm{Sm}_{2} \mathrm{Co}_{17}$ nanoparticles.

that the prepared nanoparticles have been effectively protected from oxidation during the fabrication and examination processes.

The particle size of the Sm-Co nanoparticles can be further fine tuned by controlling the settle-down time. Figure 3 shows the dependence of the particle size on settle-down time of the nanoparticles in solutions, where the particle size was analyzed by LPSA. It was observed that the particle size decreases fast with the settle-down time, especially when the settle-down time was less than $3 \mathrm{~h}$. Simultaneously, the coercivity also decreases with increasing settle-down time, i.e., with decreasing particle size. The inset of Fig. 3 shows the size dependent coercivity of the $\mathrm{Sm}_{2} \mathrm{Co}_{17}$ nanoparticles. The $3 \mathrm{~h}$ settle-down nanoparticles have coercivity of $3 \mathrm{kOe}$ at room temperature. However, the floating-at-top $\mathrm{Sm}_{2} \mathrm{Co}_{17}$ nanoparticles are superparamagnetic at room temperature and their blocking temperature was estimated to be $45 \mathrm{~K}$ by zero-field cooling and field cooling measurements (not shown here). The anisotropy of $3 \mathrm{~h}$ settle-down particles estimated by Sharrock fiting ${ }^{10}$ is about $1.7 \times 10^{5} \mathrm{erg} / \mathrm{cm}^{3}$, which is far lower than the value for bulk $\mathrm{Sm}_{2} \mathrm{Co}_{17}$ alloy ${ }^{1}$ and could be attributed to amorphorization during ball milling as mentioned above.

In order to achieve high coercivity in SmCo nanoparticles, we also scanned the composition by changing the $\mathrm{x}$ value in the $\mathrm{SmCo}_{\mathrm{x}}$ from 3.5 to 10. It has been found that the $\mathrm{SmCo}_{\mathrm{x}}$ nanoparticles with different compositions have simi-

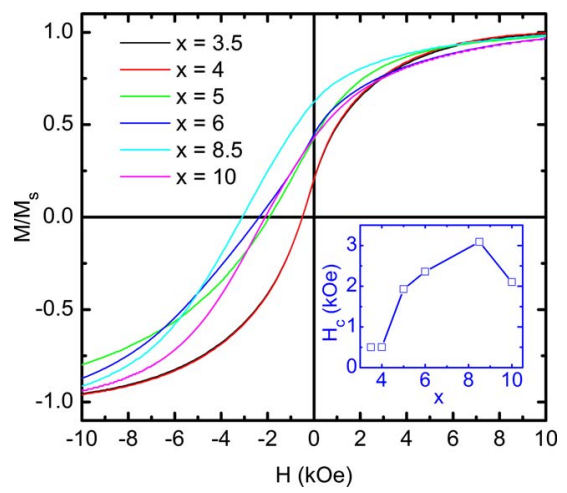

FIG. 4. (Color online) Demagnetization curves of the SmCo nanoparticles with different composition. The inset gives the composition dependent coercivity of the SmCo nanoparticles. 


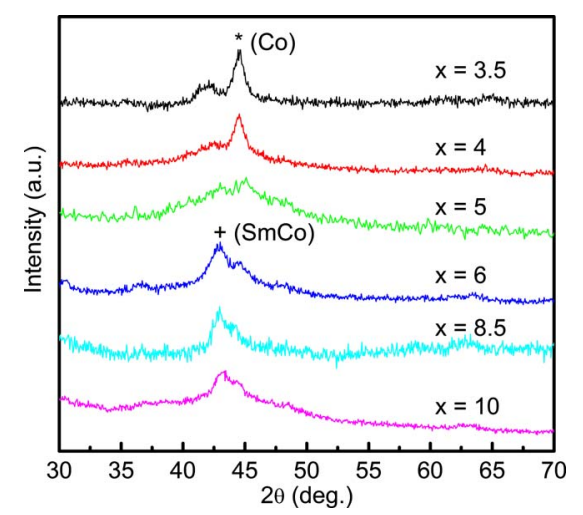

FIG. 5. (Color online) XRD patterns of SmCo nanoparticles with different compositions.

lar behavior in size selection process. Figure 4 shows the demagnetization curves of the $\mathrm{SmCo}_{\mathrm{x}}(\mathrm{x}=3.5,4,5,6,8.5$, and 10) nanoparticles obtained from particles of $3 \mathrm{~h}$ settledown, i.e., the particles size with size about $20 \mathrm{~nm}$. The room temperature coercivity of the $\mathrm{SmCo}_{\mathrm{x}}$ nanoparticles ranges from $0.5-3 \mathrm{kOe}$. As one can see from the inset of Fig. 4, the coercivity increases with increasing $\mathrm{x}$, and reaches the highest value for $\mathrm{Sm}_{2} \mathrm{Co}_{17}$ nanoparticles. Further decrease in $\mathrm{Sm}$ content also leads to the decrease in coercivity, which may be attributed to the low anisotropy in the $\mathrm{SmCo}_{10}$ alloy. The composition dependence of the coercivity can be better understood if one studies the XRD patterns of the nanoparticles. Figure 5 shows the XRD patterns of the $3 \mathrm{~h}$ settledown $\mathrm{SmCo}_{\mathrm{x}}$ nanoparticles with different composition. As one can see that the $\mathrm{Sm}_{2} \mathrm{Co}_{17}$ nanoparticles are almost single phase, while the hcp Co phase appears in the $\mathrm{SmCo}_{5}$ nanoparticles. The intensity of the Co peak, representing the amount of Co phase, increases with increasing Sm content. This is an indication that the SmCo nanoparticles with higher $\mathrm{Sm}$ content are more unstable during the milling. The similar results have also been observed in the slurry particles, decomposition of the $\mathrm{Sm}-\mathrm{Co}$ phases has taken place during the milling. This phenomenon is opposite to the anticipation that less Co content in the SmCo phases results in less free Co particles. More detailed investigation is underway.

\section{CONCLUSIONS}

In conclusion, $\operatorname{SmCo}_{\mathrm{x}}(\mathrm{x} \sim 3.5-10)$ nanoparticles with different particle size and composition have been prepared by surfactant-assisted ball milling. The coercivity of $\mathrm{SmCo}_{\mathrm{x}}$ nanoparticles was found to be dependent on the particle size and composition. In addition, the SmCo nanoparticles with low Sm content have higher stability during the process.

\section{ACKNOWLEDGMENTS}

This work was supported by U.S. DoD/MURI Grant No. N00014-05-1-0497 and DARPA through ARO under Grant No DAAD 19-03-1-0038. This work was also supported by Center of Nanostructured Materials and Characterization Center for Materials and Biology at the University of Texas at Arlington. The authors would like to express appreciations to Dr. Jinfang Liu of Electron Energy Corp. for providing the SmCo alloy powders.

${ }^{1}$ D. Weller, A. Moser, L. Folks, M. E. Best, W. Lee, M. F. Toney, M. Schwckert, J. U. Thiele, and M. F. Doerner, IEEE Trans. Magn. 36, 10 (2000).

${ }^{2}$ H. Zeng, J. Li, J. P. Liu, Z. L. Wang, and S. Sun, Nature (London) 420, 395 (2002).

${ }^{3}$ C. B. Rong, H. W. Zhang, R. J. Chen, S. L. He, and B. G. Shen, J. Magn. Magn. Mater. 302, 126 (2006).

${ }^{4}$ Y. Hou, Z. Xu, S. Peng, C. B. Rong, J. P. Liu, and S. Sun, Adv. Mater. 19, 3349 (2007).

${ }^{5}$ Y. Hou, S. Sun, C. B. Rong, and J. Ping Liu, Appl. Phys. Lett. 91, 153117 (2007).

${ }^{6}$ N. G. Akdogan, G. C. Hadjipanayis, and D. J. Sellmyer, J. Appl. Phys. 105, 07A710 (2009).

${ }^{7}$ V. M. Chakka, B. Altuncevahir, Z. Q. Jin, Y. Li, and J. P. Liu, J. Appl. Phys. 99, 08E912 (2006).

${ }^{8}$ Y. P. Wang, Y. Li, C. B. Rong, and J. P. Liu, Nanotechnology 18, 465701 (2007).

${ }^{9}$ M. Yue, Y. P. Wang, N. Poudyal, C. B. Rong, and J. P. Liu, J. Appl. Phys. 105, 07A708 (2009).

${ }^{10}$ M. P. Sharrock, J. Appl. Phys. 76, 6413 (1994). 